\title{
Meeting demand: A multi-method approach to optimizing hospital language interpreter staffing
}

\author{
Tze Chao Chiam*1, Stephen Hoover ${ }^{1}$, Danielle Mosby ${ }^{1}$, Richard Caplan ${ }^{1}$, Sarahfaye Dolman ${ }^{1}$, Adebayo Gbadebo ${ }^{1}$, \\ Frank Mayer ${ }^{1}$, Alexandra Nightingale ${ }^{1}$, Claudia-Angelica Reyes-Hull ${ }^{2}$, Elizabeth Brown ${ }^{1}$, Eric Jackson ${ }^{1}$, Bettina \\ Tweardy Riveros ${ }^{3}$, Jacqueline Ortiz ${ }^{2}$ \\ ${ }^{1}$ Value Institute, Christiana Care Health System, Newark, Delaware, United States \\ ${ }^{2}$ Department of Cultural Competence, Equity and Language Services, Christiana Care Health System, Newark, Delaware, United \\ States \\ ${ }^{3}$ Office of Health Equity, Christiana Care Health System, Newark, Delaware, United States
}

Received: January 5, 2017

DOI: $10.5430 /$ jha.v6n2p21
Accepted: February 6, 2017

Online Published: February 17, 2017

URL: https://doi.org/10.5430/jha.v6n2p21

\begin{abstract}
Objective: The objective of this paper is to highlight a study on optimizing the full-time equivalent (FTE) for Spanish and Mandarin interpreters at Christiana Care Health System. In this study, there were multiple challenges that needed to be addressed, and a multi-method approach was taken.

Methods: These methods include: (1) time-motion study to quantify interpreter workflow and variability of duration of time needed for each task; (2) an integer program to optimize the number of interpreters needed per hour based on historical demand patterns for interpreter services; (3) Discrete-Event Simulation (DES) to examine the use of agency interpreters in order to meet demand; (4) cost modelling to convert FTEs and the use of agency interpreters into overall costs to the hospital; and (5) sensitivity analysis to evaluate alternative number of interpreter FTEs and their corresponding costs to the hospital.

Results: Overall cost to the hospital is predicted to decrease with additional FTE interpreters, up to a threshold level above which the cost will start to increase. Through this innovative methodology used in this paper, we predict that hiring 3.5 more FTEs for Spanish interpreters will result in 9.07\% of cost savings, and predict that hiring one FTE for Mandarin interpreters will result in $25.87 \%$ in cost savings compared to the current expense of providing Mandarin language interpretation.

Conclusions: Contrary to intuition, increasing number of FTEs results in cost savings. Besides the financial benefit, hospitals will also be able to ensure the quality of health services that Limited English Proficiency (LEP) patients and families receive.
\end{abstract}

Key Words: Interpreters, Language services, Time-motion study, Integer programming, Discrete-Event simulation, Staffing optimization, Cost modeling

\section{INTRODUCTION}

\subsection{The United States national linguistic landscape}

Although most medical care in the United States is provided in English, a significant percentage of the population use other languages to communicate in their everyday lives. According to the 2011-2015 American Community Survey 5year estimates, $21 \%$ of Americans speak a language other than English in the home. ${ }^{[1]}$ Of these individuals, 22\% speak English not well or not at all. ${ }^{[2]}$ This amounts to over 13 mil-

\footnotetext{
*Correspondence: Tze Chao Chiam; Email: Tze.C.Chiam@ChristianaCare.org; Address: Value Institute, Christiana Care Health System, 4755 Ogletown-Stanton Road, Newark, DE 19718, United States.
} 
lion individuals nationwide who may struggle to understand their healthcare provider during routine and acute healthcare interactions. ${ }^{[2-4]}$ In Delaware, $13 \%$ of the population speaks a language other than English in the home, and $18 \%$ of these speak English not well or not at all; this is also known as Limited English Proficiency (LEP). ${ }^{[2]}$ In addition, since 1980, the number of LEP individuals has increased by $158 \%^{[2]}$ and is expected to continue to rise in the future. ${ }^{[5]}$

\subsection{Impact of interpreters on outcomes}

\subsubsection{Clinical outcomes}

LEP patients experience more adverse events than Englishspeaking patients, with $49 \%$ of LEP patients compared to $29 \%$ of English-speaking patients experiencing adverse events during a hospital stay. ${ }^{[6]}$ Language barriers are linked to decreased preventive care, lower likelihood of having a usual source of care, increased risk of infant mortality, increased risk of infectious disease, and increased risk of both chronic and acute complications. ${ }^{[4,7]}$ LEP patients are also more likely to belong to minority groups who already experience disparities in healthcare, placing them at even greater risk. ${ }^{[4,8]}$ To compensate, providers often order additional diagnostic tests, prescribe more medications, and are more likely to resort to invasive procedures. ${ }^{[4]}$

Roughly half of the adverse events experienced in the LEP population can be linked to failures in communication, compared to only a third in English-speaking patients. ${ }^{[6]}$ LEP patients who need interpretation but do not receive it report little understanding of their diagnosis and treatment plan. ${ }^{[9]}$ Use of professional interpreters can improve LEP patients' understanding and experience to nearly equal the experience of a patient speaking to a provider in their natural language. ${ }^{[10]}$ When professional interpreters are used, LEP patients are just as likely as English-speaking patients to adhere to follow-up, visit the emergency department (ED), have diagnostic tests ordered, and be appropriately admitted to the hospital. ${ }^{[10]}$ Similarly, LEP patients have equal length of stay (LOS) as English-speaking patients when professional interpreters are involved in their care. ${ }^{[10]}$ This equalization of healthcare utilization supports the use of professional interpreters in healthcare settings.

\subsubsection{Patient satisfaction}

Studies related to patient satisfaction indicate that patients prefer professional interpreters or language-concordant providers (in-person and telephonic) to ad hoc interpreters. ${ }^{[9,10]}$ While patients' preference of mode of interpretation, such as in-person or telephonic, remains unclear, patients who need interpreter services but do not receive them have the lowest satisfaction with care. ${ }^{[9]}$

\subsubsection{Cost-effectiveness}

A comparison study that examined the impact of interpreter services looked at direct costs of providing language services and secondary costs of healthcare utilization before and after a comprehensive interpreter services program was implemented in a large healthcare system. ${ }^{[1]}$ Jacobs, Shepard, Suaya, and Stone found that following the implementation of a language services program, there was a significant increase in the use of preventive services, recommended office visits, and receipt and fulfillment of prescription medications. ${ }^{[11]}$ These improvements in preventive services and office visits resulted in an increase in cost associated with provision of care. However, there was also a reduction in ED use. ${ }^{[1]}$ This improved communication may also lead to long-term reductions in the cost of care for LEP patients that were not measured in this study. ${ }^{[11]}$

\subsection{Utilization of language services}

Interpretation in healthcare settings may be provided by professional interpreters, language-concordant providers, or ad hoc interpreters, including family, friends, or other healthcare staff. ${ }^{[12]}$ Accurate medical interpreting requires command of both languages and familiarity with a broad range of medical terminology. ${ }^{[12]}$ Ad hoc interpreters are more likely to commit interpretation errors "of clinical significance" and are less likely to be trained in patient confidentiality. ${ }^{[12]}$ Despite these implications, studies of patterns of interpreter use in medical settings routinely reveal low rates of interpreter use, with physicians and nurses being more likely to rely on ad hoc interpreters than to request professional language services. ${ }^{[13]}$

\subsection{Barriers to provision of language services}

The low rates of interpreter use in the face of documented benefits of utilizing professional interpreters during healthcare encounters involving LEP patients described in section 1.3 may be related to several barriers to provision of these services. The primary barriers to providing language services during healthcare interactions are the appropriate identification of LEP patients, failure to request interpreter services, and interpreter availability.

\subsubsection{Availability of interpreters}

A qualitative study revealed that suboptimal interpreter scheduling and a limited number of available in-person interpreters are barriers to the provision of language services when they would be preferred. ${ }^{[14]}$ Many providers also "expressed dissatisfaction, distrust, and frustration with the telephonic interpretation", further limiting receipt of needed services. ${ }^{[14]}$ When language services are unavailable or when there is a time constraint, providers utilize ad hoc methods to 
communicate with patients, such as consulting the internet or smartphone translation applications, getting by with gestures, relying on untrained bilingual staff or family members, or relying on the provider's limited foreign language ability. ${ }^{[14]}$ These methods are utilized even when providers are aware of the benefit of language services and the associated legal and medical risk. ${ }^{[14]}$

\subsubsection{The lack of an interpreter staffing model}

Despite the importance of appropriately staffing hospital interpreters, unlike nurse-to-patient ratios, there are no validated models in the literature providing guidelines for optimal interpreter staffing levels. There is also a lack of published studies on understanding interpreter workflow that could be used to derive an unbiased staffing model.

\subsubsection{Use of industrial engineering and operations re- search approaches}

Optimizing staffing and scheduling is a pragmatic topic for institutions in many industries. Various methods have been used to ensure optimal staffing and scheduling. Of these methods, Operations Research is widely-used. Operations Research approaches include mathematical optimization and stochastic simulation. Labidi et al. utilized mathematical programming approach to schedule staff at a bank, ${ }^{[15]}$ Tsai et al. constructed a mathematical programming model to solve nurse scheduling problem. ${ }^{[16]}$ Stochastic simulation, such as Discrete-Event Simulation (DES) introduces probability distributions to describe the potential outcome of a random variable, hence allowing the simulation model to mimic the actual system under study for a more realistic analysis. DES has been used to predict hospital and clinic staffing, ${ }^{[17]}$ others have used such techniques to identify a staffing mix that reduces patient waiting time and increases throughput in a pharmacy. ${ }^{[18]}$

Time-motion study is a method for improving inefficiencies in the workplace by creating employee "productivity standards" by capturing employee tasks observationally. ${ }^{[19]}$ The time and motion it takes to complete daily tasks is methodically recorded by trained observers over a period of time in order to obtain an objective quantification of productivity. ${ }^{[19]}$ Time-motion study has been used to provide insights into staffing needs for various types of staff including nurses ${ }^{[20]}$ and dietitians. ${ }^{[21]}$

\subsection{Aim}

At Christiana Care Health System, the number of requests for interpreter services rose from 1,300 in January 2014 to 2,200 in October 2015, whereas the number of interpreter full-time equivalents (FTEs) remained unchanged. In order to optimize the availability of interpreters for patients

Published by Sciedu Press needing language services while simultaneously containing costs, Christiana Care Health System initiated a study that aimed to derive the number of FTE interpreters based on arrival patterns of demands for services, while taking into consideration the costs of the FTEs and the costs of utilizing contracted agency interpreters.

\section{METHOD}

\subsection{Study site}

This study was performed at Christiana Care Health System in Newark, Delaware, USA. Christiana Care is one of the largest health systems in the United States with approximately 180,000 emergency room visits annually. As the only Level-1 trauma center in the state of Delaware, Christiana Care serves a large portion of the state's population, which consists of a diverse mix of cultural and linguistic backgrounds. As Spanish and Mandarin interpretation requests accounted for $83 \%$ of total requests at the study site, this study focused on optimizing Spanish and Mandarin interpreter staffing levels.

\subsection{Conceptual model}

Current state studies revealed that in order to optimize interpreter staffing levels, a multi-method, interdisciplinary approach is needed. This approach consists of time-motion study, mathematical programming, DES, sensitivity analysis, and cost modeling. The interaction of these methods is depicted in Figure 1. Time-motion study will be used to understand current interpreter workflow and quantify duration of time needed for each activity to serve as inputs to DES. Mathematical programming will be used to derive the optimal number of interpreters needed to respond to hourly demand for services. DES will incorporate probability distribution of time duration of activities and interpreters flow path captured in the time-motion study, optimal number of interpreters from mathematical programming, as well as arrival rates of demand. Sensitivity analysis, together with cost modeling are used to study financial trade-offs between using hospital-employed interpreters and agency interpreters. The following sections describe each approach in more detail. We utilized a multi-method approach to this study; therefore this paper provides a high level overview of the methods utilized to meet the aim of the study.

\subsubsection{Time-motion study}

As interpreters are centrally-located but serve the entire hospital system, they are constantly traveling to service sites by foot or by car. In order to quantify the travel time and to capture interpreters' tasks and workflow including interpretation, we conducted a time-motion study at Christiana Hospital in Newark, Delaware, USA, over a 50-hour period with inter- 
preters who consented to participate in the study. ${ }^{[22]} \mathrm{We}$ directly observed six interpreters in an inpatient and acute care setting, excluding outpatient primary care visits. A more detailed description of the methods and results of this time-motion study can be found in Mayer et al. ${ }^{[22]}$

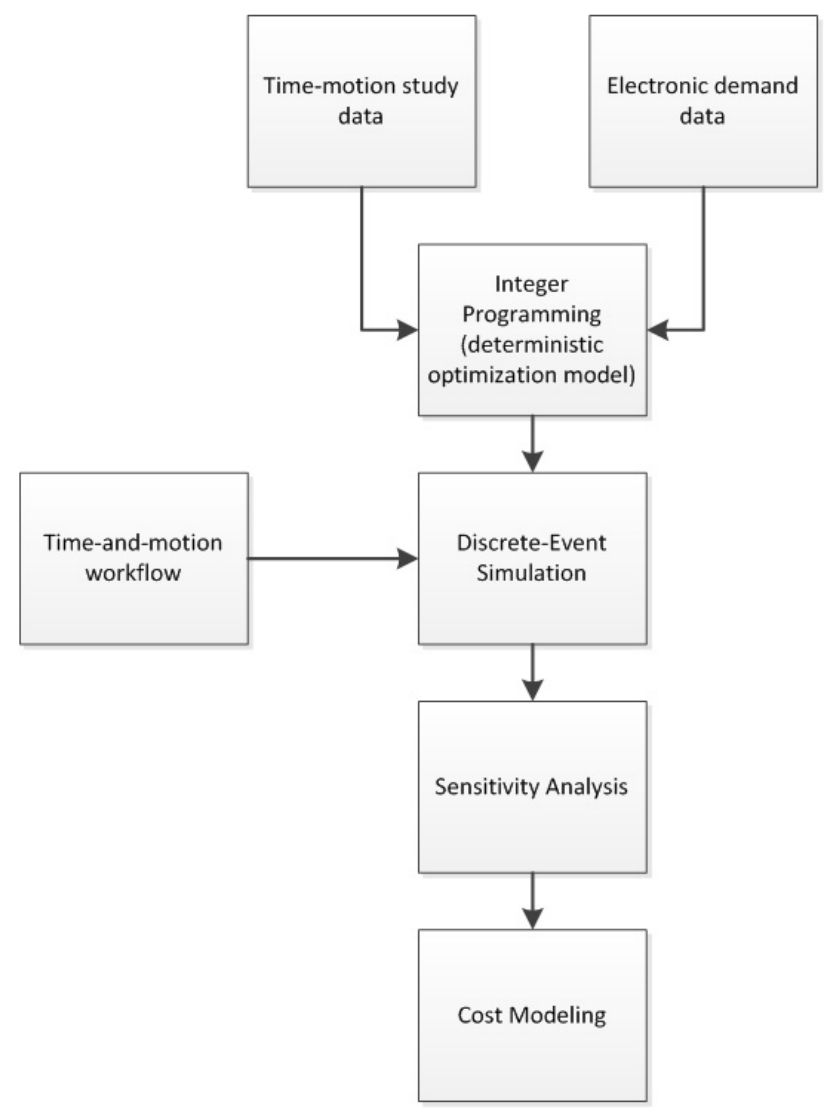

Figure 1. Conceptual model for multi-method approach for language services staffing optimization

\subsubsection{Mathematical programming}

Mathematical programming was used to find the optimal set of shift assignments for interpreters to minimize the deviation of hourly supply from hourly demand. Constraints prevent interpreters from being assigned to more than one shift per day. Further, the number of interpreters scheduled for a given hour must meet or exceed the average demand for interpretation requests at that hour. The mathematical representation is shown in equations 1 through 4 . The optimization model was solved using Microsoft Excel 2010 Solver.

Objective Function: minimize the total deviation of hourly supply from hourly demand (see Equation 1):

$$
\min \sum_{h \in H}\left(D_{h}-\sum_{j \in J_{h}} \sum_{i \in I} x_{i j}\right)
$$

Subject to Constraints: all full-time employees must be assigned to only 1 shift/day (see Equation 2):

$$
\sum_{j \in J_{F T}} x_{i j}=1, \forall i \in I_{F T}
$$

All part time employees must be assigned to only 1 shift/day (see Equation 3):

$$
\sum_{j \in J_{P T}} x_{i j}=1, \forall i \in I_{P T}
$$

Number of employees scheduled for each hour must be greater than or equal to the demand at that hour (see Equation 4):

$$
\sum_{j \in J_{H}} \sum_{i \in I} x_{i j} \geq D_{h}, \forall h \in H
$$

Where:

$\mathrm{h}=$ hour index;

$\mathrm{H}=$ set of hours in the day;

$\mathrm{D}_{h}=$ demand for interpreters at hour;

$\mathrm{j}=$ shift index;

$\mathrm{J}=$ set of shifts;

$\mathrm{i}=$ interpreter index;

$\mathrm{I}=$ set of all interpreters;

$\mathrm{I}_{F T}=$ subset of full-time interpreters;

$\mathrm{I}_{P T}=$ subset of part-time interpreters.

\subsubsection{Discrete-Event Simulation (DES)}

DES is a class of computer simulation that utilizes process flows and probability distributions to mimic and study the dynamics and interaction of various components of a complex system. DES was used in this study to predict the volume of interpreter requests being fulfilled by agency interpreters when Christiana Care's in-person interpreters are not available. The volume of requests fulfilled by agency interpreters was compared to the number of hospital-hired interpreters in order to model the trade-offs between FTEs and demand unfulfilled by hospital-employed interpreters. Input to the simulation includes demand data by time-of-day, day-of-week, languages requested, locations of service, distance, and travel time between regions of the hospital served by language services as obtained through the time-motion study; probability distribution of service duration; and the optimized number of FTEs obtained through solving the mathematical programming model outlined in section 2.2.2. Validation and verification of the simulation model were performed before the model was used for sensitivity analysis and prediction. The DES was performed using Arena Simulation v14.70. Each simulation was run for a duration of seven 
days in computer simulation time. A total of 30 replications were made for each scenario tested in order to account for variability in each simulation run.

\subsubsection{Sensitivity analysis}

Sensitivity analysis was performed to study the impact of various levels of FTEs available on volume of interpreter requests fulfilled (and unfulfilled) by hospital-employed interpreters. Sensitivity analysis was performed for Spanish fulltime FTE, Spanish per diem FTE, and Mandarin per diem FTE. Unfulfilled requests for in-person hospital-employed interpreters are assumed to be routed to contracted agency interpreters.

The base case for Spanish interpreters consists of six fulltime FTEs and one per diem FTE, which reflects the FTE configuration at the time of study. In order to allow maximum flexibility of scheduling interpreters, the six full-time FTEs were kept constant, and the number of per diem FTEs needed to reach optimum was studied. Hence, sensitivity analysis will show the cost outcome as the number of per diem FTEs increases from one to nine. The base case for Mandarin interpreters consists of 0.5 per diem FTE. Sensitivity analysis will show the cost outcome as the number of per diem FTEs increases from 0.5 to 2 .

\subsubsection{Cost model}

A cost model was constructed based on the variable costs of hospital-employed interpreters as well as the variable costs of utilizing agency interpreters. This cost model (see Equation 5) does not include sunk costs, capital investment, equipment, office space, administrative salaries, or any other costs not directly incurred with additional FTEs.

$$
T C=s_{f} x_{1}+s_{p} x_{2}+C\left[\sum_{k=1}^{2}\left(r_{k} t_{k} d_{k}\right)+R(130)\right]
$$

Where:

$T C=$ Total cost;

$s_{f}=$ average salary of a full-time interpreter (including fringe benefits);

$x_{1}=$ number of full-time interpreters;

$s_{p}=$ average salary of a per diem interpreter;

$x_{2}=$ number of per diem interpreters;

$C=$ total predicted volume (from DES) of requests for interpreters;

$k=1$ for requests sent to vendor $1 ; 2$ for requests sent to vendor 2;

$r_{k}=$ number of calls to agencies for phone or video interpretation;

$t_{k}=$ hourly cost of utilizing agencies for phone or video interpretation;

$d_{k}=$ duration (in hours) of utilizing agencies for phone or Published by Sciedu Press video interpretation;

$R=$ number of requests to agencies for in-person interpretation.

Agencies providing in-person interpretation require a 2-hour minimum pay at a rate of $\$ 65 /$ hour, hence every request for such agencies is assumed to incur a cost of $\$ 130$, as indicated in Equation 5.

Demand for utilizing agency interpreter services as predicted through DES was converted into the cost of utilizing agency interpreters through cost modelling. The number of hospitalemployed FTEs was converted to FTE cost. Due to the sensitive nature of cost data, actual costs are not disclosed in this paper. Instead, cost ratios are used to illustrate the outcomes of the study. The cost ratio for a scenario is calculated by dividing the total cost associated with that scenario by the lowest total cost of all the scenarios studied. As a result, the scenario with the lowest cost will have a cost ratio of 1 . All other scenarios will have a cost ratio of greater than or equal to 1 .

\subsection{Data sources}

\subsubsection{Vendor database}

In order to analyze historical data regarding demand for interpreter services, data were extracted from a third party vendor database. Data elements extracted included patient identifiers, start date and time of service requested, end date and time of service requested, location (unit and room) of service performed, language requested, and status of request for all interpreter requests received between January 1, 2014 and September 30, 2015.

\subsubsection{Time-motion study}

In order to perform in-person interpretation services, interpreters must travel from their base location to the service location or from a previous service location to the next service location. This travel time was taken into consideration while optimizing staffing as it is an essential activity in order for the service to be performed. The flow paths of interpreters were also quantified to serve as input to the DES.

\section{RESULTS}

\subsection{Time-motion study}

Analysis shows that during the period of direct observation, interpreters spent time on 18 different activities (see Table 1). Interpreting for patients, waiting (for patients and/or providers), traveling (to/from service location), attending meetings, waiting for test results, and waiting through interruptions accounted for $80 \%$ of interpreter time observed during the study period. Total time per activity indicates the summation of duration in minutes that was spent engaging 
in each activity during the entire observation period $(3,000$ minutes). Count reflects the number of times an activity was observed during the entire observation period.

\subsection{Mathematical programming}

The deterministic scheduling model indicates that six fulltime interpreters and at least eight part-time interpreters are necessary in order to meet demand. Solutions with less than eight part-time interpreters were infeasible due to the inability to satisfy the constraint for hourly demand. The solved model produced a matrix of the binary scheduling assignments for each interpreter. These scheduling assignments were then used in the DES for validation, sensitivity, and cost analysis.

\subsection{DES, sensitivity analysis, and cost model}

Sensitivity analysis shows that as the number of Spanish interpreter FTEs increases from the base case, the number of requests to agencies decreases and the cost ratio also decreases. The cost ratio continues to decrease up to 10.5 FTEs, after which it increases with additional increase of FTEs. Therefore, 10.5 FTEs will result in a predicted cost savings of $9.07 \%$, as shown in Table 2 (Scenario 2) and Figure 2.

Sensitivity analysis of Mandarin interpreter FTEs shows a similar trend. Mandarin interpreter FTEs are optimized at 1 FTE with a corresponding predicted cost savings of $25.87 \%$, as shown in Table 3 (Scenario 1) and Figure 3.

Table 1. Activities and corresponding statistics from time-motion study ${ }^{[22]}$

\begin{tabular}{llllll}
\hline Activity & Total time per activity (min) & Percent & Count & Mean & Standard Deviation \\
\hline Interpreting for Patients & 968.37 & 32.97 & 111 & 8.72 & 7.76 \\
Waiting (for patients and/or providers) & 518.10 & 17.64 & 40 & 12.95 & 10.5 \\
Traveling (for/from providing service) & 327.35 & 11.15 & 72 & 4.55 & 3.19 \\
Meetings & 206.50 & 7.03 & 10 & 20.65 & 18.43 \\
Waiting for Test Results & 180.32 & 6.14 & 24 & 7.51 & 6.11 \\
Interruptions & 150.57 & 5.13 & 55 & 2.92 & 3.22 \\
Input for Database & 145.22 & 4.94 & 52 & 2.79 & 5.54 \\
Dispatch & 110.63 & 3.77 & 3 & 36.88 & 50.46 \\
Translating & 107.55 & 3.66 & 7 & 15.36 & 14.68 \\
Consult & 75.83 & 2.58 & 46 & 1.65 & 1.70 \\
Interpreting for Non-patients & 41.20 & 1.40 & 13 & 3.17 & 2.86 \\
Travel-Canceled & 33.22 & 1.13 & 11 & 3.02 & 1.19 \\
Video Remote Interpretation & 15.67 & 0.53 & 2 & 7.83 & 1.85 \\
Education & 15.22 & 0.52 & 7 & 2.17 & 1.82 \\
Personal Protective Equipment & 12.83 & 0.44 & 11 & 1.17 & 1.06 \\
Incident Report & 11.83 & 0.40 & 1 & 11.83 & 0.00 \\
Other Cancelled Activities & 11.48 & 0.39 & 4 & 2.87 & 1.85 \\
Looking for Supplies & 4.93 & 0.17 & 2 & 2.47 & 1.78 \\
\hline
\end{tabular}

Table 2. Outcomes (cost ratios) from DES, sensitivity analysis, and cost modeling for Spanish interpreters

\begin{tabular}{lllllll}
\hline & Base case & Scenario 1 & Scenario 2 & Scenario 3 & Scenario 4 & Scenario 5 \\
\hline Full-time FTE & 6 & 6 & 6 & 6 & 6 & 6 \\
Per diem FTE & 1 & 4 & 4.5 & 5.5 & 6.5 & 9 \\
Calls to agencies & 308 & 235 & 228 & 218 & 208 & 196 \\
Cost ratio & 1.0997 & 1.0029 & 1.0000 & 1.0044 & 1.0089 & 1.0532 \\
\hline
\end{tabular}

Note. FTE: full-time equivalent

\section{Discussion}

The use of professional language interpreters in healthcare has been shown to provide positive outcomes. Despite such evidence, and despite the growing diversity in cultures and languages in the population of the United States, there are var- ious barriers to the provision of language services in healthcare settings. The barrier investigated in this study is the ability for hospitals to provide an optimal number of interpreters to satisfy the demand for these services. This study focused on optimizing the FTEs for Spanish and Mandarin interpreters at Christiana Care Health System. 


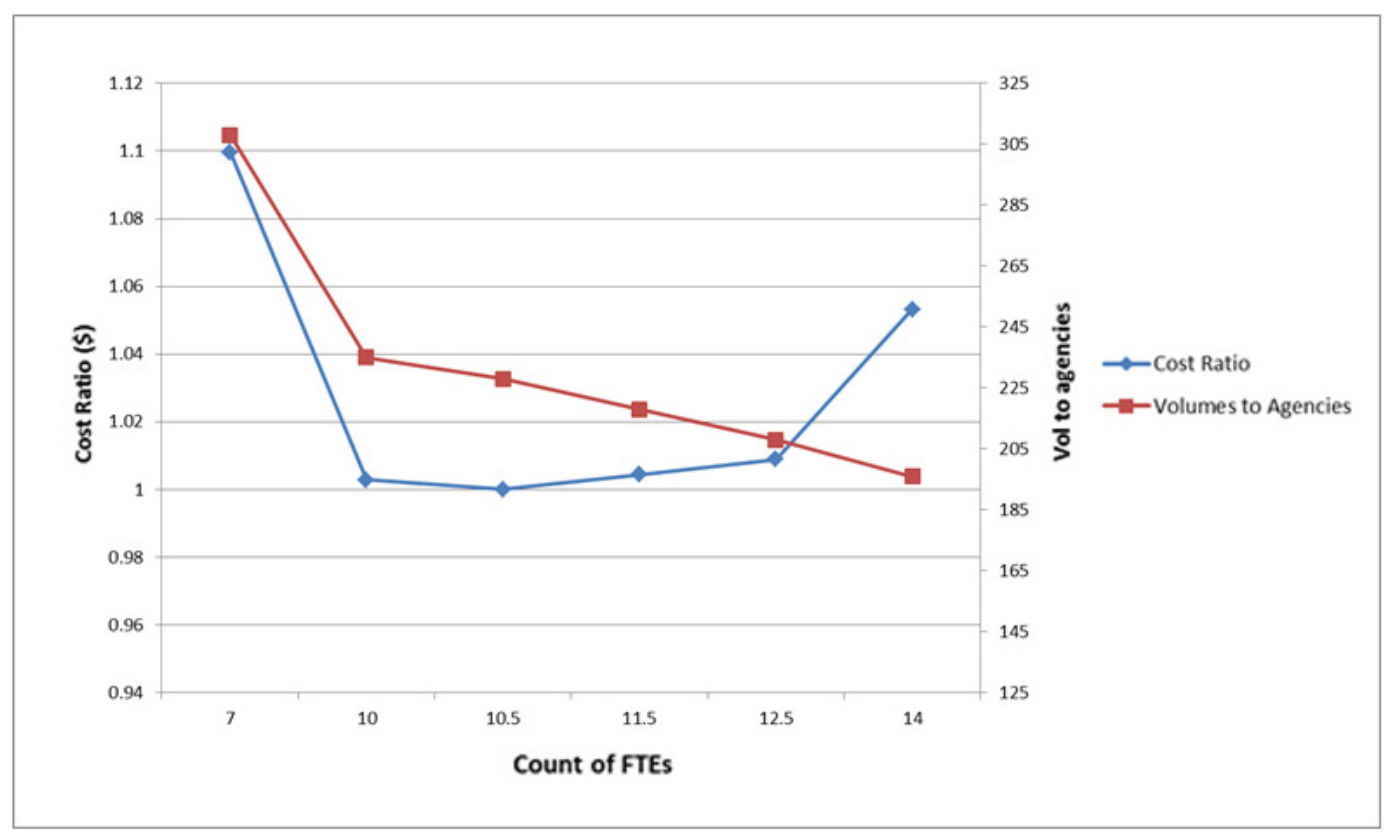

Figure 2. Trade-off between Spanish interpreter FTEs and volumes and cost

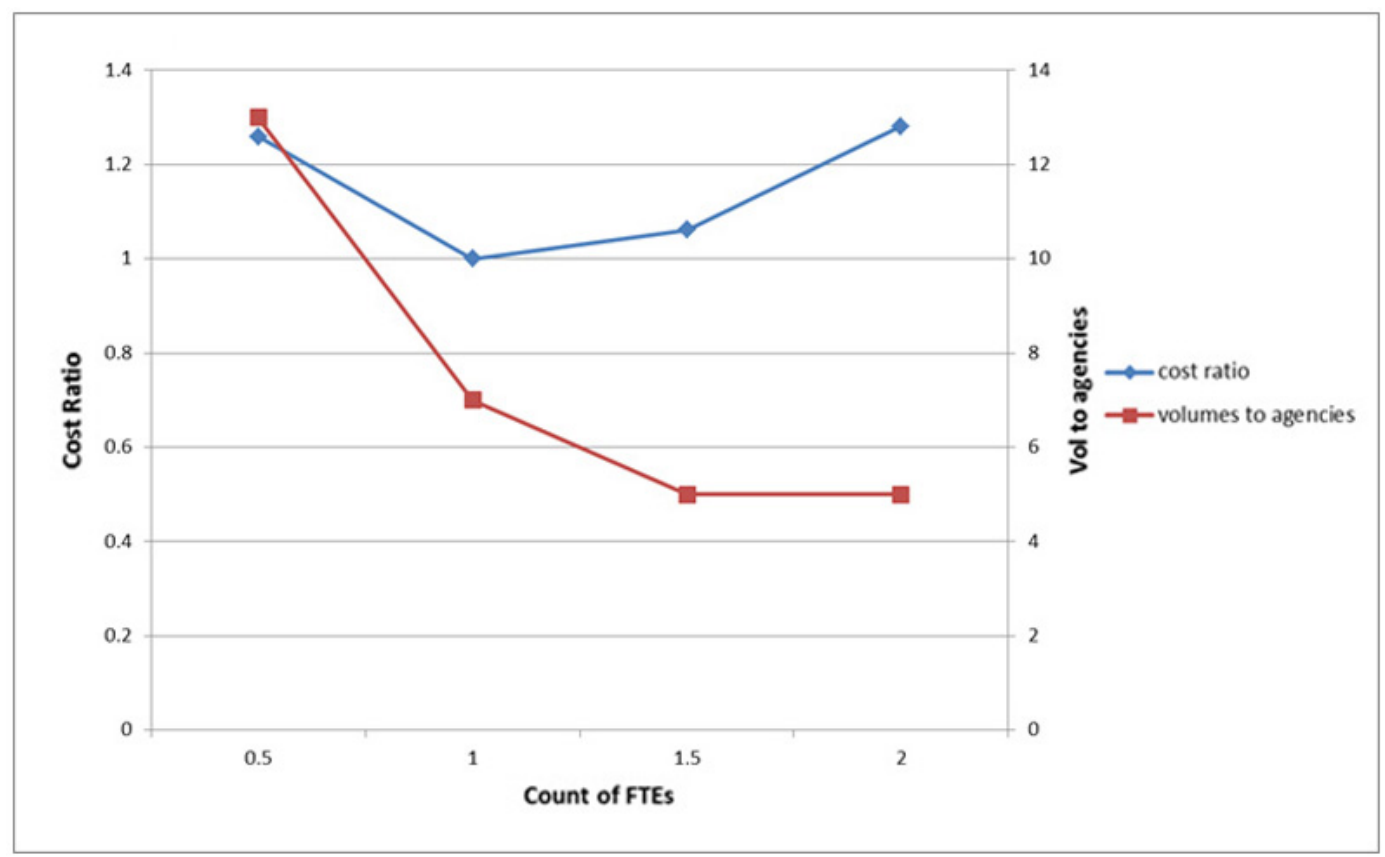

Figure 3. Trade-off between Mandarin interpreter FTEs and volumes and cost

Table 3. Outcomes (cost ratios) from DES, sensitivity analysis, and cost modeling for Mandarin interpreters

\begin{tabular}{lllll}
\hline & Base case & Scenario 1 & Scenario 2 & Scenario 3 \\
\hline Full-time FTE & 0 & 0 & 0 & 0 \\
Per diem FTE & 0.5 & 1 & 1.5 & 2 \\
Calls to agencies & 13 & 7 & 5 & 5 \\
Cost ratio & 1.2587 & 1.0000 & 1.0608 & 1.2813 \\
\hline
\end{tabular}

Note. FTE: full-time equivalent

Published by Sciedu Press
Results from our analyses are counterintuitive. Contrary to the general belief that hiring additional staff will incur higher costs to the hospital, our analysis showed that overall cost to the hospital is predicted to decrease with additional FTEs, up to a threshold level above which the cost will start to increase. This threshold level for Spanish interpreters at Christiana Care is 10.5 FTEs. As the current FTE count of 
Spanish interpreters is 7 , we predict that hiring 3.5 additional FTEs will result in $9.07 \%$ cost savings based on the current expense on Spanish language interpretation. Similar analysis showed that the optimal level of Mandarin interpreters is 1 FTE, where it is currently 0.5 FTE. The increase to 1 FTE is projected to result in $25.87 \%$ cost savings compared to the current expense of providing Mandarin language interpretation. The observed results can be attributed to the current under-staffing of hospital-employed Spanish and Mandarin interpreters, high cost of agency interpreters, as well as variability of demands for interpretation services.

Besides the financial benefit, hospitals which have their own language services department will also be able to ensure the quality of interpreters by obtaining feedback from providers and patients regarding services they receive from interpreters and providing training and monitoring of interpreter performance.

\section{LIMITATIONS AND FUTURE WORK}

Although this study provided insight into utilizing a multimethod approach to optimizing hospital interpreter staffing and demonstrated the financial benefits of this optimization, there are a number of limitations and proposed work for future studies.

\subsection{Quantification of clinical outcomes}

This study focused on the financial trade-off between utilizing both hospital-employed interpreters and agency interpreters. However, previous studies have shown evidence that utilizing utilization of professional interpreters results in improved clinical outcomes in addition to financial benefits. Quantifying financial savings from clinical outcomes such as prevention of medical errors, reduced utilization of hospital resources, and prevention of invasive and expensive tests performed would provide a more comprehensive analysis of overall cost savings that is not captured in the scope of the current study. As language services are often viewed by hospital administrators as a "cost center", such quantification will also provide information on the true value that such services provide for the hospital.

\subsection{Time-motion study}

While the time-motion study provided useful insights to interpreter activity, it represented only a 50-hour snapshot of interpreter workflow in the inpatient care units on one campus. A future study could be conducted to provide insights into workflow of interpreters and quantification of interpreters' task duration in outpatient clinics, emergency departments, as well as potentially non-patient care areas in other campuses that the interpreters currently serve.

\subsection{Other considerations}

As the role of language interpreters continues to evolve, interpreters are assigned additional responsibilities such as translating patient education materials and providing patient education sessions at hospital discharge. Such activities are not taken into consideration in this study as they are currently under development at Christiana Care. As a result, the staffing optimization model was constructed to optimize FTEs without such responsibilities included. A future model could be developed to allow for flexibility of incorporating additional responsibilities in order to calculate appropriate level of staffing and provide improved levels of service to patients and providers.

Additionally, assessing the impact of interpreter services on patient satisfaction for LEP patients who received interpreter services and those who did not should be addressed in the future.

\section{ACKNOWLEDGEMENTS}

This work was initiated by the Christiana Care Finance department and the Language Services department. The authors of this paper would like to acknowledge Thomas Corrigan, Robert McMurray and William Conway from Finance for their support of this work. The authors also like to acknowledge the interpreters in the Language Services department for their consent on participation in the time-motion study as part of the overall study.

\section{CONFLiCTS OF INTEREST Disclosure}

The authors declare they have no conflict of interest.

\section{REFERENCES}

[1] 2011-2015 American Community Survey: "S1601: Speak a language other than English" [Internet]. United States Census Bureau, American Community Survey Office. 2015 [cited 2016 Dec 10]. Available from: http://factfinder. census.gov

[2] Ryan C. Language use in the United States: 2011. Washington, DC: US Census Bureau; 2013.

[3] Wilson E, Chen AH, Grumbach K, et al. Effects of limited English proficiency and physician language on health care comprehension. J Gen Intern Med. 2005; 20(9): 800-6. PMid: 16117746. PMid: 16117746. https://doi.org/10.1111/j.1525-1497.2005.0 $174 . \mathrm{x}$

[4] Regenstein M, Huang J, West C, et al. Hospital language services: quality improvement and performance measures. In Henrikson $\mathrm{K}$, Battles JB, Keyes MA, Grady ML (Eds). Advances in Patients Safety: New Directions and Alternative Approaches (Vol. 2: Culture and 
Redesign). Rockville, MD: Agency for Healthcare Research and Quality; 2008. PMid: 21249903.

[5] Ortman JM, Shin HB. Language projections: 2010 to 2020. Paper presented at American Sociological Association Annual Meeting: Caesar's Palace, Las Vegas, NV; Aug 20-23, 2011.

[6] Divi C, Koss RG, Schmaltz SP, et al. Language proficiency and adverse events in US hospitals: a pilot study. Int J Qual Health Care. 2007; 19(2): 60-7. PMid: 17277013.

[7] Regenstein M, Andres E. Hospital language service programs: a closer look at translation practices. J Health Care Poor Underserved. 2014; 24(4): 2003-18. PMid: 25418255. https ://doi .org/10.1 $353 / \mathrm{hpu} .2014 .0162$

[8] Jacobs EA, Lauderdale DS, Meltzer D, et al. Impact of interpreter services on delivery of health care to limited-English-proficient patients. J Gen Intern Med. 2001; 16(7): 468-74. PMid: 11520385. https : //doi.org/10.1046/j.1525-1497.2001.016007468.x

[9] Flores G. The impact of medical interpreter services on the quality of health care: a systematic review. Med Care Res Rev. 2005; 62(3): 255-99. PMid: 15894705. https://doi.org/10.1177/107755 8705275416

[10] Karliner LS, Jacobs EA, Chen AH, et al. Do professional interpreters improve clinical care for patients with limited English proficiency? A systematic review of the literature. Health Serv Res. 2007; 42(2): 727-54. PMid: 17362215. https://doi.org/10.1111/j.1475 $-6773.2006 .00629 . \mathrm{x}$

[11] Jacobs EA, Shepard DS, Suaya JA, et al. Overcoming language barriers in health care: costs and benefits of interpreter services. Am J Public Health. 2004; 94(5): 866-9. PMid: 15117713. https : //doi.org/10.2105/AJPH.94.5.866

[12] Vanderwielen LM, Enurah AS, Rho HY, et al. Medical interpreters: improvements to address access, equity, and quality of care for limited-English-proficient patients. Acad Med. 2014; 89(10): 13247. PMid: 25054413. https://doi.org/10.1097/ACM. 000000 0000000296

[13] Schenker Y, Pérez-Stable EJ, Nickleach D, et al. Patterns of interpreter use for hospitalized patients with limited English profi- ciency. J Gen Intern Med. 2011; 26(7): 712-7. PMid: 21336672 https ://doi.org/10.1007/s11606-010-1619-z

[14] Michalec B, Maiden KM, Ortiz J, et al. Providers' perceptions of medical interpreter services and limited English proficiency (LEP) patients: understanding the "bigger picture". J Appl Soc Sci. 2015; 9(2): 156-169. https://doi.org/10.1177/1936724414550247

[15] Labidi M, Mrad M, Gharbi A, et al. Scheduling IT staff at a bank: a mathematical programming approach. Scientific World J. 2014: 768374. PMid: 24772032. https://doi.org/10.1155/2014/7 68374

[16] Tsai CC, Lee CJ. Optimization of nurse scheduling problem with a two-stage mathematical programming model. Asia Pacific Management Rev. 2010; 15(4): 503-16. Available from: http: //apmr .man agement.ncku.edu.tw/comm/updown/DW1012213789.pdf

[17] DeRienzo CM, Shaw RJ, Meanor P, et al. A discrete event simulation tool to support and predict hospital and clinic staffing. Health Informatics J. 2016 [Epub ahead of print]. PMid: 26928193. https://doi.org/10.1177/1460458216628314

[18] Mukherjee AK. Simulation model for management of operations in the pharmacy of a hospital. SIMULATION. 1991; 56(2): 91-103. https://doi.org/10.1177/003754979105600205

[19] Lopetegui M, Yen PY, Lai A, et al. Time motion studies in healthcare: what are we talking about? J Biomed Inform. 2014; 49: 292-9. PMid: 24607863. https://doi.org/10.1016/j·jbi.2014.02.017

[20] Hendrich A, Chow MP, Skierczynski BA, et al. A 36-hospital time and motion study: how do medical-surgical nurses spend their time? Perm J. 2008; 12(3): 25-34. PMid: 21331207. https : //doi.org/10.7812/TPP/08-021

[21] Hand RK, Jordan B, DeHoog S, et al. Inpatient staffing needs for registered dietitian nutritionists in 21 st century acute care facilities. $\mathrm{J}$ Acad Nutr Diet. 2015; 115(6): 985-1000. https ://doi .org/10.1 $016 / j \cdot j$ and .2015 .01 .013

[22] Mayer FC, Mosby DL, Nightingale A, et al. Quantifying medical interpreter activity: a time-motion study. Del J Public Health. 2016; 2(5): 22-26. 